\title{
Channel capacity maximization using NQHN approach at heterogeneous network
}

\author{
Savita Patil ${ }^{1}$, A. M. Bhavikatti ${ }^{2}$ \\ ${ }^{1}$ AMC Engineering College, India \\ ${ }^{2}$ Computer Science and Engineering, BKIT, India
}

\begin{tabular}{l} 
Article Info \\
\hline Article history: \\
Received Jun 30, 2018 \\
Revised Feb 7, 2019 \\
Accepted Mar 9, 2019 \\
\hline
\end{tabular}

\section{Keywords:}

Base stations (BSs) Optimized traffic scheduling (OTS) Wireless communication (WC) Quality-of-service (QoS) Novel QoS aware HetNets (NQHN)

\section{Corresponding Author:}

Savita Patil,

AMC Engineering College,

Bangalore-83, India.

Email: sampati1949@gmail.com

\begin{abstract}
In present scenario, the high speed data transmission services has pushed limits for wireless communication network capacity, at same time multimedia transmission in real-time needs provision of QoS, therefore the network capacity and small cell coverage has comes with lots of challenges. Improving the channel capacity and coverage area within the available bandwidth is necessary to provide better QoS to users, and improved channel capacity for the FCUs and MCUs in network. In this paper, we are proposing an NQHN approach that incorporate with efficient power allocation, improving the channel capacity by optimized traffic scheduling process in a small cell HetNets scenario. This work efficiently handle the interference with maintaining the user QoS and the implemented power controller uses HeNB power as per the real time based approach for macro-cell and femtocell. Moreover, we consider the real traffic scenario to check the performance of our proposed approach with respect to existing algorithm.
\end{abstract}

Copyright () 2019 Institute of Advanced Engineering and Science. All rights reserved.

\section{INTRODUCTION}

HetNets are a future generation WC networks that have been used to reduce the unsighted area of the mobile communication with improving the present network coverage area in compared with traditional WC networks. A WAN (Wide Area Network) can use macro-cell and, femto-cell or pico-cell to provide the wide coverage area in a wireless coverage environment such as; homes, office buildings, underground areas and an open outdoor area. The usage of mobile data are growing exponentially through several type of communication applications like as; multimedia phones and, Wi-Fi etc. It is not possible to satisfy the larger communication requirements like as coverage and throughput using the traditional WC network by macrocell BSs (Base Stations).

Moreover, to provide the novel applicant methodology in LTE-A based WC networks, the HetNets has been propose in [1-3] that enhances the data rate and network area coverage. In HetNets, there are several low-energy and low-cost femto-cell are distributed around the macro-cell BSs, said to be as femto-cell users, which shares the same available spectrum bandwidth with the macro-cell users in order to get optimized spectral efficiency in a cellular network. Therefore, the interference from the users of femto-cell to macrocell BSs should be monitor and control strictly, also the mitigation of interference is very necessary for the control power based 'resource allocation' and used as practical approach in wireless HetNets [4, 5]. The resource allocation approach for HetNets has concern from many researchers and its importance is growing extremely, the major aim in 'resource allocation' for existing femto-cell networks is to decrease the received interference at macro-cell users, simultaneously achieve the femto-cells performance from using power control approach that has been studied in [6-8]. 
In paper [6], they proposed an approach of interference mitigation in order to enhance the uplink throughput via providing a stable interference threshold value, also through regulating transmit power of femto-cell user. The two-tier femto-cell system has considered in [7], where resource allocation has provided in both uplink and downlink for enhancing the sensitivity capacity of femto-cells users, decreasing the delay timing at femto-cell users under macro-cell user interference constraint and quality service constraint for sensitive users. In paper [8], they proposed an energy efficient HetNets by using game theory at resource allocation process in a downlink transmission under a multichannel HetNets. It is point to be considered that the mostly approach related to resource allocation required perfect knowledge of CSI (i.e., channel state information) at transmitter side, however, it is generally considered that all system knowledge such as perfect-CSI are accessible to femto-users, due to arbitrary behavior of incorrect channel estimation, channel delays and wireless channels. Therefore, it is difficult for femto-users to acquire the desirable system parameter values such as; interference power and channel gains from different networks. In paper [9, 10], they proposed a robust optimization approach that exhibits robustifying resource allocation with imperfectCSI, which has drawn significant attention in order to handle the uncertainty in HetNets. The major aim of power controller is to minimalize the power in transmission, therefore decrease the high power consumption and reducing the inter-cell interferences in necessary as we discussed previously. Through properly regulating the downlink power transmission as per resource block is necessary to get achievable bit-rate in femto-cells, all interference that generated in small cell network can be reduce significantly.

Therefore improving the channel capacity and coverage area within available bandwidth is necessary to provide better quality of service to users, though protecting macro-cell users in network through maintaining the interference under a threshold level. The effectiveness QoS at traffic users is also key factor and without any provision, the level of QoS can be mishandled in LTE-A (long-term evolution advanced) based small network. Moreover, the increment in mobile users causes the degradation in QoS, due to its more data usage (i.e., more bandwidth) applications. In this paper, we are proposing an NQHN approach that incorporate with efficient power allocation, improving the channel capacity by optimized traffic scheduling process in a small cell HetNets scenario. This work efficiently handle the interference with maintaining the user QoS, the implemented power controller uses HeNB power as per the real time based approach for macro-cell and femto-cell. Moreover, the power controller approach uses 3GPP [11] standard for dynamic representation of efficient 'power switching' points and optimized traffic scheduling (OTS) approach to perform QoS aware scheduling by considering traffic parameters with real-time HetNets condition. In result section, we consider the number of femto-cell user and macro-cell users in a traffic scenario to check the performance of our proposed approach and providing comparison analysis with existing algorithm.

\section{LITERATURE SURVEY}

In order to face the traffic related issues in WC networks, it is necessary to coordinate and utilize the several large throughput 'small-cell' like as wireless LAN (local area networks). Moreover, the number of large throughput 'small-cell' has considered in [12], where they constructed the small outdoor cells via access set up points at indoors. To validate the system performance, the indoor-outdoor field measurement has done in order to propagate in multiple direction; also, they focused on $3.5 \mathrm{GHz}$ that used in small-cell of LTE-A system. In this paper [13], they used tool such name as stochastic geometry, also designed a framework model for the downlink data-rate coverage probability in a small cell network with enabling MIMO at wireless backhaul. The small cell network is consist of several small cells, which can configured either in out-band and in-band types of backhaul under an assured probability. The user performance has consider in hierarchical network and limited through several interferences sources such as; small-cell BS interference, backhaul interfaces, etc. The effect of channel difficulty under MIMO and wireless backhaul faces long-term channel arrangement, where the access link involved in both long and short term of channel effects.

The general grid approach has become stubborn as per the increasing in network size, also it cannot handle the structure of outgeneral networks, therefore it is become challenging to compute the accurate performance of WC cellular network, because of propagation effect in path and network prototype complexity. Therefore, a way should be there in order to simulate the cellular networks and in [14], the several network model was compared by simulation. However, estimating the performance of network via simulation can deliver understanding on specific setting thus the outcome may not differ at other scenarios as well as the computational complexity, in [15] they also proposed the work based on cellular network enhancement with fixed approach. Their proposed approach has efficiently work to achieve the optimum result at a small-cell HetNets, while considering the large HetNets with this approach may create more complexity. Furthermore, the cooperation with sophisticated BS and local 'or' global CSI are required to get output of achievable performance under a communal network setting.

Int J Elec \& Comp Eng, Vol. 9, No. 4, August 2019 : 2593 - 2602 
The application of WC dense devices and services access required high consumption of energy, due to real time processing, for that energy efficient design has consider for financial and environment cause. Therefore, it become trend to find out best energy efficient process and as per our information, generally OFDM is used in small cell HetNets to provide power allocation, higher energy efficiency, bandwidth allocation in wireless backhaul, and user QoS. Where the QoS is novel approach for this field, which investigated less and in paper [16], they proposed an energy efficient allocation technique for wireless backhaul network that based on OFDM access HetNets small cell. There are also some existing technique of resource allocation, which increases the throughput and increases the efficiency of energy through allocating dual transmit power level at individual small-cell BS to users and channel bandwidth, that based on circuit power ingestion and CSI. The present backhaul networks consist of statically resource allocation that result little allocations when the several small-cells are present in a cellular network with given resources, therefore, in [17] they proposed new access backhaul network design that based on Smart-GW (Gateway) in between BSs and small-cell. Specifically, they applied modest LTE protocol, which add the Smart-GW into advanced LTE HetNets.

In paper [18], they proposed a random spatial methodology where base stations are modified as spatial PPP (Poisson point process), these type of random network topology has widely used in wireless ad-hoc network [19-22] and it has performed well under small cell network scenario where the position of BS are in irregular form. In paper [23], they proposed LAA ('licensed-assisted access') for the investigation of small cell network and a framework called LTE with unlicensed incumbent model has introduced here, where they give expression for both transmission strategies; wireless fidelity (Wi-Fi) and LTE system under an unlicensed spectrum. In [24-26], the point process has consider with the stochastic geometry theory, this methodology shows the appropriate and tractable performance that can used to examine the throughput and probability in cellular networks. In addition, a random spatial network approach can be used in different type of network such as distributed antenna structures [27] and HetNets [28-31], but from the above study, we have adopted that still a lag in optimizing the HetNets performance with maintaining the user QoS.

\section{PROPOSED METHODOLOGY}

Here, we consider femto-cells that has ability to avoid the interference with different channel signals; also, deliver high quality data transmission to mobile users, therefore femto-cells enhances the spectral efficiency at number of user per unit coverage area. Moreover, the BS present at shorter distance, which help mobile terminals to get much energy efficiency through decreasing the transmission power and that, increases the battery life. The use of femto-cells at indoor location, the macro-cells can also provide much reliable service to outdoor users because of the overhead reduction. Figure 1 shows the proposed model block diagram, which shows two major part such as power controller approach and optimized traffic scheduling algorithm in a real-time streaming scenario with maintain users QoS, the QoS at heterogeneous network dynamically considered for the users. In HetNets scenario, femto-cells users and macro-cells users are makes request, for that acquired channel state and traffic information are forwarded to scheduling and power controller process, so that we can achieve optimized trans-receiver BS (TBS) and user throughput.

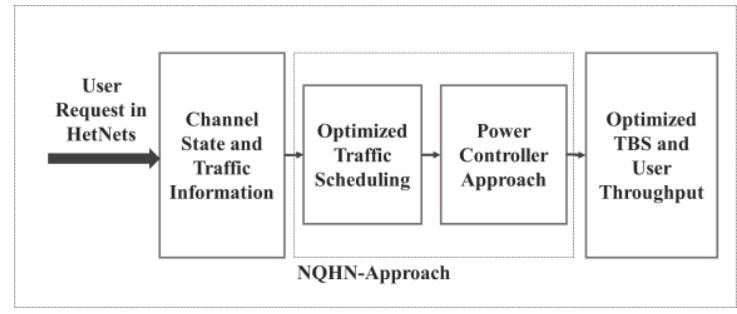

Figure 1. Block Diagram of Proposed NQHN Approach

\subsection{Optimized traffic scheduling (OTS) algorithm}

In this section, we describe the optimized scheduling algorithm in order to handle the traffic occurrence effectively in a small-cell HetNets, also provide acceptable capacity to a system. The acquired channel state and traffic information are given input to OTS algorithm to make the scheduling result at a period of time, which also based on utility computation function [32]. The utility function aim is obtain the standardized QoS objective that realized through user network scenario and in general, the packet holding time of a user are high so the requirement of QoS also become more for that user. 


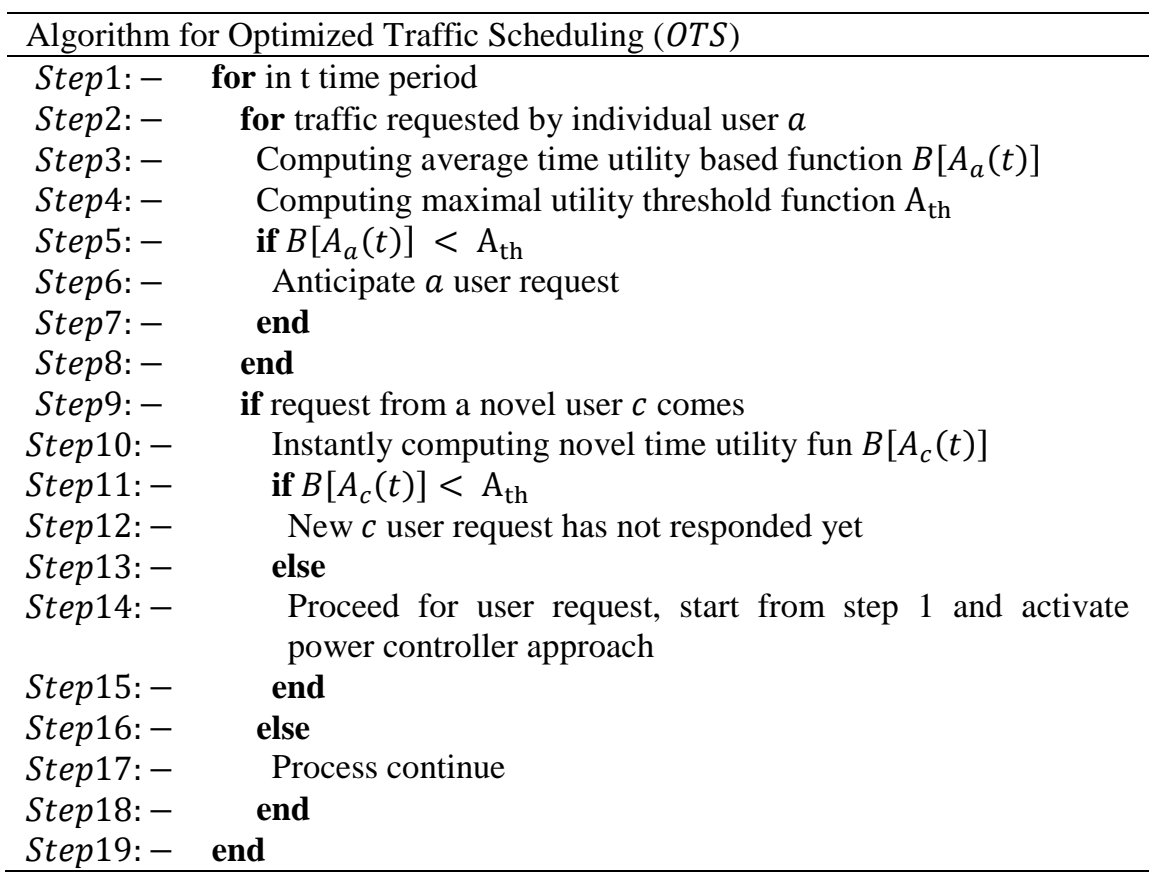

Moreover, the QoS has provided in controller multimedia transmission and, for real-time scenario, we can use any data transmission so that the delay in performance may occurs. The delay and throughput performance are major in lower priority users but it is not much critical, due to regulating the angle of delay bounds that can vary utility functional metric instantly. In addition it is found that the above OTS algorithm has achieve better performance in a period when the users number are not very large and the femto-cell users move closely towards BS in HetNets. The user movement and handover request distant from the femto-cell center needs more 'load balancing', which causes falls in system capacity and the performance services.

\subsection{Robust user quality based power controller}

A multiuser OFDM based HetNets is considered which contains $D$ number of femto-cell users (FCUs) and communicating with associated femto-cell BSs (FCBSs) over $E$ number of subcarrier. FCUs are used to utilize the macro-cell users (MCUs) via FC-BSs, where $D$ and $E$ are varies according to active user's number and available subcarrier, that can be indexed as;

$$
\begin{aligned}
& d \in \mathfrak{D} \triangleq\{1,2,3 \ldots \ldots, D\} \\
& e \in \mathcal{E} \triangleq\{1,2,3 \ldots \ldots, E\}
\end{aligned}
$$

Here, we assumed that $\mathcal{E} \geq \mathfrak{D}$, the subcarrier bandwidth is assumed to be $F \mathrm{~Hz}$ that is very less compare to the wireless channel bandwidth, therefore applying Shannon Hartley Theorem (SHT) [33] corresponding FCU data rate $d$ at subcarrier $e$ is written as.

$$
g_{d, e}=F h_{d, e} \log _{2}\left(1+I_{d, e} J_{d, e} / K_{d, e}\right)
$$

Where, $K_{d, e}$ denotes the $d$ FCU background noise at $e$ subcarrier, $h_{d, e}$ denotes the $d$ FCU subcarrier assignment at $e$ subcarrier, $I_{d, e}$ denotes the $d$ FCU transmit power at $e$ subcarrier and $J_{d, e}$ denotes the $d$ FCU direct channel gain at $e$ subcarrier. The subcarrier assignment will be 0 or 1 that shows the $e$ subcarrier is used by $d$ FCU or not. The major constraint is battery capacity at $m$ th FCU transmitter and the individual FCU can use limited amount of power, therefore the constraint is given as;

$$
\sum_{e=1}^{E} h_{d, e} I_{d, e} \leq I_{d}^{\max }, \forall d \in \mathfrak{D}
$$

In (4), $I_{d}^{\max }$ denotes the maximal power transmit of FCU and the data-rate should fulfil the minimal requirement of $d$ FCU QoS that written as;

$$
\sum_{e=1}^{E} g_{d, e} \geq G_{d}^{\min }, \forall d \in \mathfrak{D}
$$


where, $G_{d}^{\min }$ shows the minimal requirement rate of $d \mathrm{FCU}$ and the interference constraint of total cross-tier under femtocell networks to the MCU receiver part can be described as;

$$
\sum_{d=1}^{D} \sum_{e=1}^{E} h_{d, e} I_{d, e} N_{d, e} \leq M^{i l}
$$

where, the interference level at MCU receiver is denote by $M^{i l}$ and the maximization of sum rate via power controller at HetNets can be given as;

$$
\begin{aligned}
& \max _{h_{d, e} I_{d, e}} \sum_{d=1}^{D} \sum_{e=1}^{E} g_{d, e} \\
& \sum_{e=1}^{E} h_{d, e}=1, \forall d \in \mathfrak{D}, Z_{1} \\
& \sum_{k=1}^{K} h_{d, e} I_{d, e} \leq I_{d}^{\max }, \forall d \in \mathfrak{D}, Z_{2}
\end{aligned}
$$

where, $Z_{1}$ shows the individual $e$ subcarrier that assigned to each FCU, $I_{d, e}=1$ signify the $e$ th-subcarrier that used by $d \mathrm{FCU}$, and $Z_{2}$ shows the power transmission constraint of $d$ FCU over the subcarrier.

$$
\sum_{e=1}^{E} G_{d, e} \geq G_{d}^{\min }, \forall d \in \mathfrak{D}, Z_{3}
$$

Equation (8) ensure the QoS for individual FCU,

$$
\begin{aligned}
& \sum_{d=1}^{D} \sum_{e=1}^{E} h_{d, e} I_{d, e} N_{d, e} \leq M^{i l}, Z_{4} \\
& h_{d, e} \in\{0,1\}, \forall d \in \mathfrak{D}, e \in \mathcal{E}, Z_{5}
\end{aligned}
$$

Where, $Z_{4}$ shows the total power interference at MCU receiver side, the major difficulty is $h_{d, e}=1$ is mixed integer and non-convex programming difficulty and $N_{d, e}$ shows the channel gains feedback that provided by MCU to FCU. In current development, mostly of the researchers has focused on power allocation strategy in HetNets [34] that focus on enhancement power with considering perfect CSI [35]. In particle, the present of quantization errors and estimation error causes the channel uncertainty that is harmful for MCUs and, in order to decrease that, we should consider some advancement technique, which can deal with these uncertainties. Therefore, here we use robust user quality based power controller and, the (8) and (9) can be rewritten in the probability form such as;

$$
\begin{aligned}
& \max _{h_{d, e} I_{d, e}} \sum_{d=1}^{D} \sum_{e=1}^{E} g_{d, e} \text { s.t. } Z_{1}, Z_{2}, Z_{5} \\
& \mathrm{P}\left\{\sum_{e=1}^{E} g_{d, e} \leq G_{d}^{m i n}\right\} \leq Q_{d}, \forall d \in \mathfrak{D}, Z_{6} \\
& \mathrm{P}\left\{\sum_{d=1}^{D} \sum_{e=1}^{E} h_{d, e} I_{d, e} N_{d, e}>M^{i l}\right\} \leq \jmath
\end{aligned}
$$

where, both (10) and (11) ensure the MCU and FCU QoS via using the probability function and $z$ and $Q_{d}$ shows the threshold value of outage probability. Here, OFDM feature technique has consider, so there the subcarrier are independent from each other and each FCU data are mutually independent from all subcarrier and the set of data-rate is defined as;

$$
\begin{gathered}
S^{e}=\left\{g_{d, e} \leq G_{d}^{\min }\right\}, \\
S=\left\{\sum_{e=1}^{E} g_{d, e} \leq G_{d}^{\min }\right\}
\end{gathered}
$$

where, $S$ set is an intersection subset of $S^{e}$ such as;

$$
\bar{S} \subset S=S^{1} \cap S^{2} \ldots S^{e} .
$$

After applying the probability analysis, we got following relationship;

$$
\{\bar{S}\} \leq \mathrm{P}\{S\}=\prod_{e=1}^{E} \mathrm{P}\left\{S^{e}\right\}
$$


Further, it can be written as;

$$
\mathrm{P}\left\{\sum_{e=1}^{E} g_{d, e} \leq G_{d}^{\min }\right\} \leq \prod_{e=1}^{E} \mathrm{P}\left\{g_{d, e} \leq G_{d}^{\min }\right\}
$$

The probabilistic rate constraint for upper bound should satisfies the required outage probability during the worst scenario, therefore the (10) can be written as;

$$
\operatorname{Max} \mathrm{P}\left\{\sum_{e=1}^{E} g_{d, e} \leq G_{d}^{\min }\right\} \leq \prod_{e=1}^{E} \mathrm{P}\left\{g_{d, e} \leq G_{d}^{\min }\right\} \leq Q_{d}
$$

In order to provide deterministic outage probability the above (17) can be written as;

$$
G_{d}^{\text {min }} \leq F h_{d, e} \log _{2}\left(1+\frac{I_{d, e}}{K_{d, e}} \mathrm{~J}_{J_{d, e}}^{-1}\left(Q_{d} / E\right)\right), \forall d \in \mathfrak{D}
$$

The satisfaction of above (18) ensure the power transmission with the considered outage probability, similarly, the probabilistic interference (11) can be modified as;

$$
h_{d, e} I_{d, e} \leq \frac{M^{i l}}{E \mathrm{~N}_{N_{d, e}}^{-1}(\sqrt[D E]{1-3})},, \forall d \in \mathfrak{D}, \forall e \in \mathcal{E} .
$$

Therefore, the (19) equation said to be deterministic and it is require to keep it as presentable, moreover, the power controller difficulty without any information can be represented as;

$$
\begin{aligned}
& \max _{d, e} I_{d, e} \sum_{d=1}^{D} \sum_{e=1}^{E} g_{d, e} \text { s.t. } Z_{1}, Z_{2}, Z_{5} \\
& F h_{d, e} \log _{2}\left(1+\frac{h_{d, e}}{K_{d, e}} \mathrm{~J}_{J_{d, e}}^{-1}\left(Q_{d} / E\right)\right) \geq G_{d}^{\text {min }}, d \in \mathfrak{D} .
\end{aligned}
$$

Here, we have applied the inverse collective distribution function at variable such as $J_{d, e}$ and $N_{d, e}$, and those can be written as $\mathrm{J}_{J, e}^{-1}$ and $\mathrm{N}_{N_{d, e}}^{-1}$.

$$
E h_{d, e} I_{d, e} \mathrm{~N}_{N_{d, e}}^{-1}(\sqrt[D E]{1-3}) \leq M^{i l}
$$

Generally, the FCUs can acquire the CSI through the channel estimation in between FCUs and MCUs, so these can cause some difficulty at CSI acquisition. Therefore, here we consider the independent model of Gaussian distribution to handle the uncertainty parameters. Moreover, the channel gain from the FCUs transmitter to BS is acquire via a robust user-quantizer and the feedback is given back to corresponding FCUs transmitter.

\section{RESULT ANALYSIS}

In this section, we presented the simulated results that is simulated in Matlab 2016b environment and the system configuration; Intel i5 processor, 2GB NVidia graphics-card, 8GB RAM and Windows 10 OS (Operating System). Moreover, we consider the several necessary parameters that generally used in traffic condition scenarios; gain of antenna $14 \mathrm{dBi}$, maximum and minimum transmit power are $20 \mathrm{dBm}$ and $0 \mathrm{dBm}$, transmit power of BS $43 \mathrm{dBm}$, speed of users $3 \mathrm{Km} / \mathrm{h}$, Urban type channel model, correlation distance $40 \mathrm{~m}$, radius of cell $1 \mathrm{Km}$, carrier and subcarrier bandwidth $2000 \mathrm{Mhz}$ and $375 \mathrm{KHz}$, system bandwidth $10 \mathrm{MHz}$ and etc.

With considering these traffic parameters, we have taken 1 macro-cell, 10 femto-cell, 15 number of MCUs, 60 subcarrier and 100 number of FCUs, and the location of femto-cell, MCUs and FCUs are generated randomly. Here, Figure 2 represents the proposed network prototype and further we will focus on 4, 7, 8 and 10. The inputs of femto cells were selected arbitrary under real-time scenario such as video data [36] and audio [37] to provide realistic multimedia transmission. The increment of mobile users will trigger additional signal interference at FCUs and MCUs in small cells scenario. 


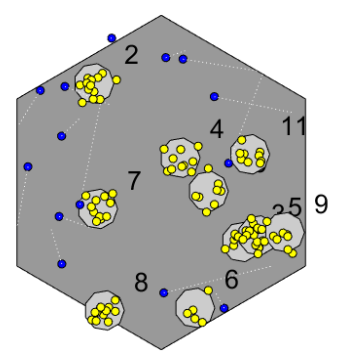

Figure 2. Proposed Network Prototype

Figure 3 shows the transmission power that used by different algorithm in cell 4 , where the existing algorithm HARQ-CC [38] and HARQ-T1 [39] has used average power of 16.52dBm and 20dBm, where our propose model NQHN has used $14.33 \mathrm{dBm}$ average power that is $28 \%$ lesser compare to HARQ-T1 [39] and $13.25 \%$ lesser compare to HARQ-CC [38].

Figure 4 shows the computed throughput by different algorithm in cell 4 , where the existing algorithm HARQ-CC [38] and HARQ-T1 [39] has obtained average throughput of 124 Mbps and 88.69 Mbps, where our propose model NQHN has got $135 \mathrm{Mbps}$ average throughput that is $7.9 \%$ more compare to HARQ-CC [38] and 34\% more compare to HARQ-T1 [39].

Figure 5 shows the transmission power that used by different algorithm in cell 7, where the existing algorithm HARQ-CC [38] and HARQ-T1 [39] has used average power of 12.2dBm and 17.16dBm, where our propose model NQHN has used $10.35 \mathrm{dBm}$ average power that is $39 \%$ lesser compare to HARQ-T1 [39] and $15.14 \%$ lesser compare to HARQ-CC [38].

Figure 6 shows the computed throughput by different algorithm in cell 7, where the existing algorithm HARQ-CC [38] and HARQ-T1 [39] has obtained average throughput of $120 \mathrm{Mbps}$ and $99.7 \mathrm{Mbps}$, where our propose model NQHN has got $142 \mathrm{Mbps}$ average throughput that is $15.4 \%$ more compare to HARQ-CC [38] and $29.8 \%$ more compare to HARQ-T1 [39].

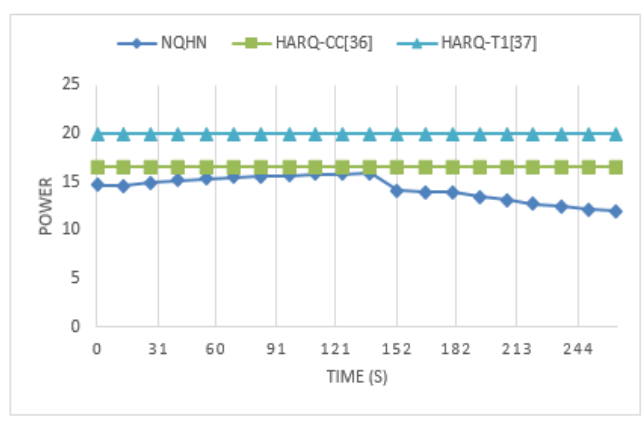

Figure 3. Power $(\mathrm{dBm})$ in Cell 4

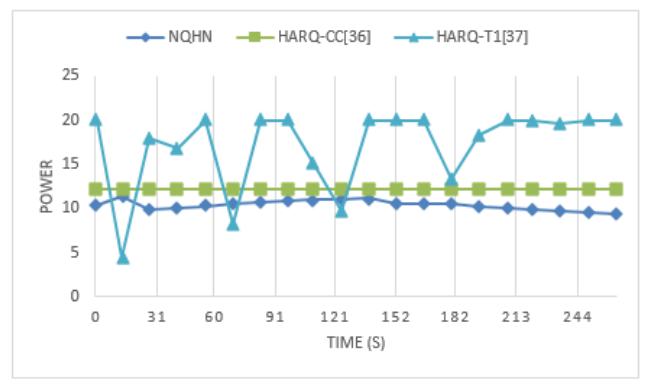

Figure 5. Power $(\mathrm{dBm})$ in Cell 7

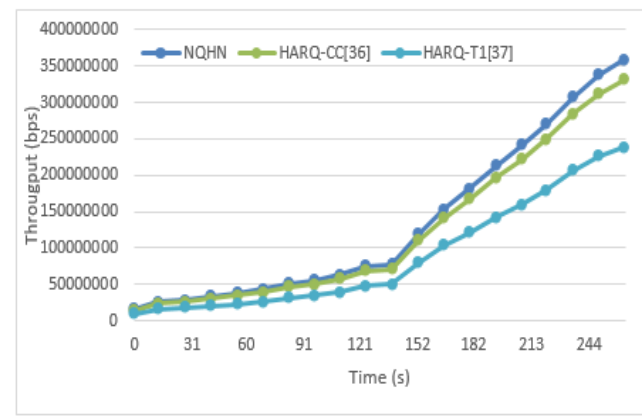

Figure 4. Throughput (bps) in Cell 4

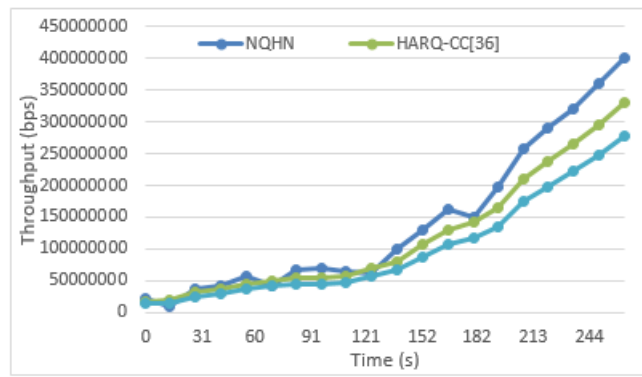

Figure 6. Throughput (bps) in Cell 7 
The transmission power used in cell 8 by different algorithm has shown in Figure 7, where, the average power used by proposed NQHN is $8.44 \mathrm{dBm}$, which is $1 \%$ more compare to HARQ-CC [38] and 46.5\% less compare to HARQ-T1 [39]. Moreover, the throughput in Mbps are obtained by different algorithm in cell 8 has shown in Figure 8, where, the average throughput of our proposed approach is 142 Mbps that is $29 \%$ more compare to HARQ-CC [38] and 27.6\% more compare to HARQ-T1 [39].

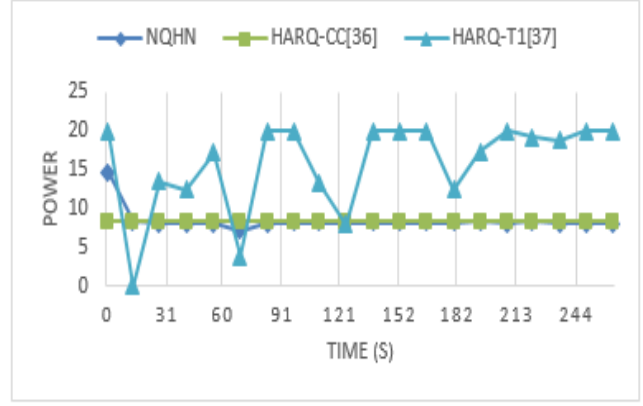

Figure 7. Power $(\mathrm{dBm})$ in Cell 8

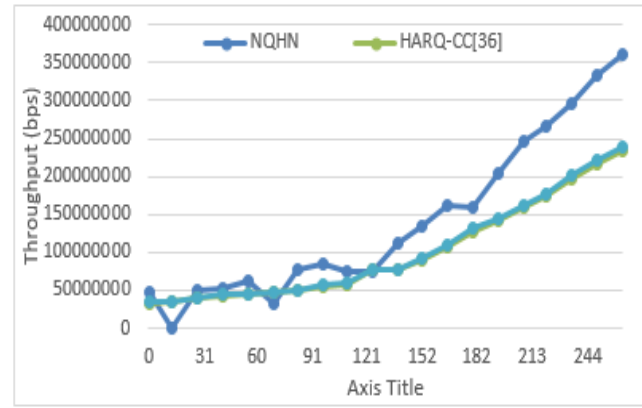

Figure 8. Throughput (bps) in Cell 8

Similarly, Figure 9 shows the transmission power that used by different algorithm in cell 10 where, the average power used by proposed NQHN is $12.43 \mathrm{dBm}$, which is $12.3 \%$ lesser compare to HARQ-CC [38] and 32.66\% less compare to HARQ-T1 [39]. Moreover, Figure 10 shows the computed throughput by different algorithm in cell 10 where, the average throughput of our proposed approach is $134 \mathrm{Mbps}$, which is $12 \%$ more compare to HARQ-CC [38] and 32.7\% more compare to HARQ-T1 [39].

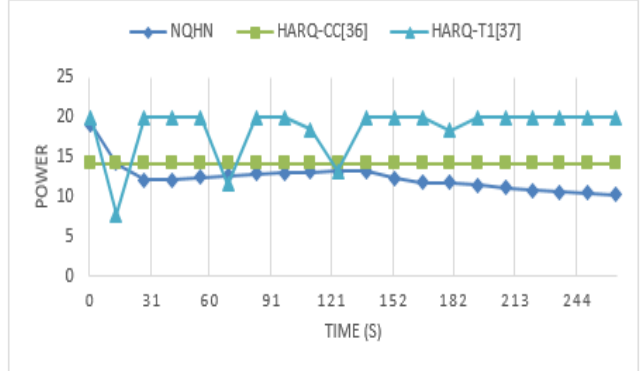

Figure 9. Power $(\mathrm{dBm})$ in Cell 10

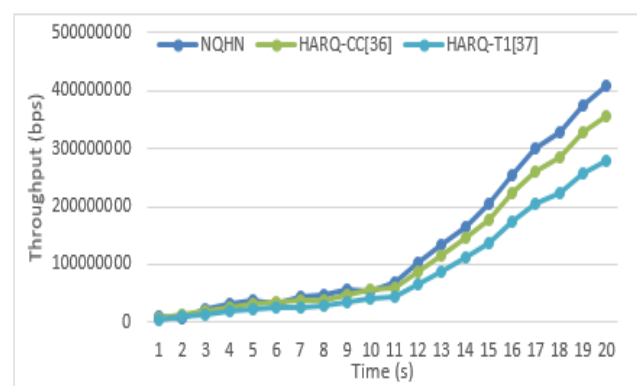

Figure 10. Throughput (bps) in Cell 10

Figure 11 shows the average throughput of considered HetNets, where our proposed approach got 25 Mbps, HARQ-CC [38] got $22 \mathrm{Mbps}$ and HARQ-T1 [39] got 19 Mbps throughput rate. Moreover, Figure 12 shows the computed delay from different algorithm in end-to-end considered HetNets scenario, where NQHN got $0.5 \mathrm{sec}$ of delay, which is $61 \%$ less delay compare to HARQ-CC [38] and $90 \%$ less compare to HARQ-T1 [39].

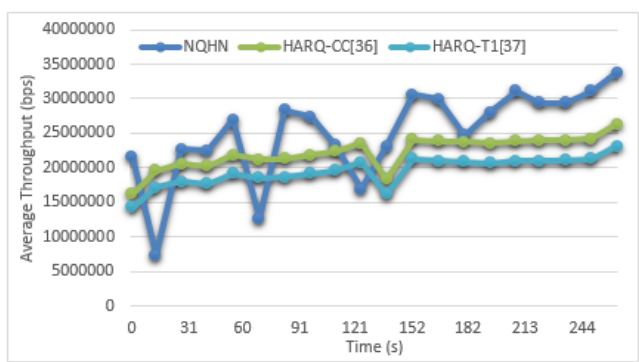

Figure 11. Average Throughput (bps)

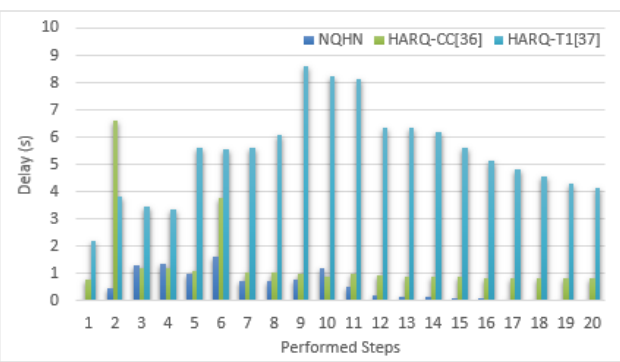

Figure 12. Computed Delay from different Algorithm 


\section{CONCLUSION}

The traffic and QoS related issues in WC networks are growing continuously. Therefore, it is necessary build the small outdoor cells (i.e., macro-cell) by setup the access points (i.e., femto-cell). In this paper, we proposed Novel QoS aware HetNets (NQHN), which contains OTA and robust user quality based power controller in order to provide QoS of macro-cell HetNets and improve system capacity. The optimized scheduling algorithm has used in order to handle the traffic occurrence effectively in a small-cell HetNets that also provide acceptable capacity to a system. The acquired channel state and traffic information are given input to OTS algorithm to make the scheduling result at a period. Moreover, the quantization errors and estimation error causes the channel uncertainty that is harmful for MCUs and for that we consider the robust user quality based power controller. In result section, we have shown sum rate maximization for a two-tier HetNets with multiple femto-cells and one macro-cell, where our proposed approach has got $11 \%$ more throughput compare to HARQ-CC [38] and 22\% more throughput compare to HARQ-T1 [39], which channel capacity enhancement by our proposed model.

\section{REFERENCES}

[1] B. Balavenkatesh, et al., "Enhancement of QoS of VOIP over Heterogeneous Networks by Improving Handoff Speed and Throughput," 2009 International Conference on Advances in Computing, Control, and Telecommunication Technologies, Trivandrum, Kerala, pp. 840-844, 2009.

[2] A. Umer, et al., "Coverage and Rate Analysis for Massive MIMO-Enabled Heterogeneous Networks with Millimeter Wave Small Cells," 2017 IEEE 85th Vehicular Technology Conference (VTC Spring), Sydney, NSW, pp. $1-5,2017$.

[3] Z. Liu and Y. Ji, "Intercell Interference Coordination under Data Rate Requirement Constraint in LTE-Advanced Heterogeneous Networks," 2014 IEEE 79th Vehicular Technology Conference (VTC Spring), Seoul, pp. 1-5, 2014.

[4] W. Xia, et al., "Large System Analysis of Resource Allocation in Heterogeneous Networks with Wireless Backhaul," IEEE Transactions on Communications, vol/issue: 65(11), pp. 5040-5053, 2017.

[5] W. Xia, et al., "Energy-efficient task scheduling and resource allocation in downlink C-RAN," 2018 IEEE Wireless Communications and Networking Conference (WCNC), Barcelona, Spain, pp. 1-6, 2018.

[6] H. S. Jo, et al., "Interference mitigation using uplink power control for two-tier femtocell networks," IEEE Trans. Wirel. Commun, vol. 8, pp. 4906-4910, 2009.

[7] H. J. Zhang, et al., "Resource allocation in spectrum-sharing OFDMA femtocells with heterogeneous services," IEEE Trans. Commun, vol. 62, pp. 2366-2377, 2014.

[8] T. Mao, et al., "Distributed energy-efficient power control for macrofemto networks," IEEE Trans. Veh. Technol, vol. 65, pp. 718-731, 2016.

[9] A. M. Abdelhady, et al., "Energy-Efficient Resource Allocation for Phantom Cellular Networks with Imperfect CSI," IEEE Transactions on Wireless Communications, vol/issue: 16(6), pp. 3799-3813, 2017.

[10] A. Ben-Tal and A. Nemirovski, "Selected Topics in Robust Convex Optimization," Math. Program, vol. 112, pp. 125-158, 2007.

[11] F. Rezaei, et al., "LTE PHY performance analysis under 3GPP standards parameters," 2011 IEEE 16th International Workshop on Computer Aided Modeling and Design of Communication Links and Networks (CAMAD), Kyoto, pp. 102-106, 2011.

[12] H. Fukudome, et al., "Measurement of $3.5 \mathrm{GHz}$ Band Small Cell Indoor-Outdoor Propagation in Multiple Environments," European Wireless 2016; 22th European Wireless Conference, Oulu, Finland, pp. 1-6, 2016.

[13] H. Tabassum, et al., "Analysis of Massive MIMO-Enabled Downlink Wireless Backhauling for Full-Duplex Small Cells," IEEE Transactions on Communications, vol/issue: 64(6), pp. 2354-2369, 2016.

[14] E. Kurniawan and A. Goldsmith, "Optimizing cellular network architectures to minimize energy consumption," Proc. 2012 IEEE Int. Conf. Commun, 2012.

[15] E. Bj"ornson and E. Jorswieck, "Optimal resource allocation in coordinated multi-cell systems," Found. Trends Commun. Inf. Theory, vol/issue: 9(2-3), pp. 113-381, 2013.

[16] H. Zhang, et al., "Downlink Energy Efficiency of Power Allocation and Wireless Backhaul Bandwidth Allocation in Heterogeneous Small Cell Networks," IEEE Transactions on Communications, vol/issue: 66(4), pp. 1705-1716, 2018.

[17] A. S. Thyagaturu, et al., "SDN-Based Smart Gateways (Sm-GWs) for Multi-Operator Small Cell Network Management," IEEE Transactions on Network and Service Management, vol/issue: 13(4), pp. 740-753, 2016.

[18] J. G. Andrews, et al., "A tractable approach to coverage and rate in cellular networks," IEEE Trans. Commun, vol/issue: 59(11), pp. 3122-3134, 2011.

[19] S. P. Weber, et al., "Transmission capacity of wireless ad hoc networks with outage constraints," IEEE Trans. Inf. Theory, vol/issue: 51(12), pp. 4091-4102, 2005

[20] F. Baccelli, et al., "An Aloha protocol for multihop mobile wireless networks," IEEE Trans. Inf. Theory, vol/issue: 52(2), pp. 421-436, 2006.

[21] A. M. Hunter, et al., "Transmission capacity of ad hoc networks with spatial diversity," IEEE Trans. Wireless Commun, vol/issue: 7(12), pp. 5058-5071, 2008.

[22] R. H. Y. Louie, et al., "Open-loop spatial multiplexing and diversity communications in ad hoc networks," IEEE Trans. Inf. Theory, vol/issue: 57(1), pp. 317-344, 2011. 
[23] P. R. Li and K. T. Feng, "Energy-Efficient Channel Access for Dual-Band Small Cell Networks," GLOBECOM 2017 - 2017 IEEE Global Communications Conference, Singapore, pp. 1-6, 2017.

[24] D. Stoyan, et al., "Stochastic Geometry and Its Applications," Wiley, 1987.

[25] F. Baccelli and B. Błaszczyszyn, "Stochastic Geometry and Wireless Networks," Now Publishers Inc., 2009.

[26] M. Haenggi, et al., "Stochastic geometry and random graphs for the analysis and design of wireless networks," IEEE J. Sel. Areas Commun, vol/issue: 27(7), pp. 1029-1046, 2009.

[27] J. Zhang and J. Andrews, "Distributed antenna systems with randomness," IEEE Trans. Wireless Commun, vol/issue: 7(9), pp. 3636-3646, 2008.

[28] V. Chandrasekhar and J. Andrews, "Spectrum allocation in tiered cellular networks," IEEE Trans. Commun, vol/issue: 57(10), pp. 3059-3068, 2009.

[29] W. C. Cheung, et al., "Throughput optimization, spectrum allocation, and access control in two-tier femtocell networks," IEEE J. Sel. Areas Commun, vol/issue: 30(3), pp. 561-574, 2012.

[30] D. Cao, et al., "Optimal base station density for energyefficient heterogeneous cellular networks," Proc. 2012 IEEE Int. Conf. Commun, 2012

[31] H. Dhillon, et al., "Modeling and analysis of K-tier downlink heterogeneous cellular networks," IEEE J. Sel. Areas Commun, vol/issue: 30(3), pp. 550-560, 2012.

[32] I. F. Chao and C. S. Chiou, "An enhanced proportional fair scheduling algorithm to maximize QoS traffic in downlink OFDMA systems," 2013 IEEE Wireless Communications and Networking Conference (WCNC), Shanghai, 2013.

[33] O. Rioul and J. C. Magossi, "On Shannon's Formula and Hartley's Rule: Beyond the Mathematical Coincidence," Entropy, vol/issue: 16(9), pp. 4892-4910, 2014.

[34] A. H. Arani, et al., "Distributed learning for energy efficient resource management in selforganizing heterogeneous networks," IEEE Trans. Veh. Technol, vol. 66, pp. 9287-9303, 2017.

[35] H. J. Zhang, et al., "Downlink energy efficiency of power allocation and wireless backhaul bandwidth allocation in heterogeneous small cell networks," IEEE Trans. Commun, vol. 95, pp. 1-13, 2017.

[36] P. Seeling and M. Reisslein, "Video Transport Evaluation with H.264 Video Traces," IEEE Communications Surveys \& Tutorials, vol/issue: 14(4), pp. 1142-1165, 2012.

[37] C. Bouras, et al., "A simulation framework for lte-a systems with femtocell overlays," ACM New York, NY, USA, 2012.

[38] M. Sheng, et al., "Performance Analysis of Heterogeneous Cellular Networks with HARQ under Correlated Interference," IEEE Transactions on Wireless Communications, vol/issue: 16(12), pp. 8377-8389, 2017.

[39] Evolved Universal Terrestrial Radio Access (E-UTRA), "Radio Resource Control (RRC)," Protocol Specification, document TS 36.331, Rev. 12.5.0, 3GPP, 2015. 